\title{
Steve White: A Hero for Patients with Epilepsy
}

\author{
Susan Axelrod ${ }^{1}[$
}

Received: 18 January 2017 / Accepted: 20 January 2017 / Published online: 14 February 2017

(C) Springer Science+Business Media New York 2017

In 1981, my daughter Lauren developed idiopathic, intractable epilepsy. She was seven months old.

And so I entered the world of epilepsy-along with the family members of 65 million people worldwide. Not by choice, but because this very unwelcome intruder-epilepsy - had entered our lives with a vengeance and with no warning. Its impact has been devastating-it robbed Lauren of much of her childhood as well as her ability to live independently as an adult.

In 1998, after over twenty different medications in multiple combinations, vagal nerve stimulation, ketogenic dietary therapy and surgery had all failed to control her seizures, I joined forces with other mothers to spearhead the search for a cure, and founded Citizens United for Research in Epilepsy (http://www.CUREepilepsy.org).

At that point, I entered the world of epilepsy willingly and with a passion driven by our own experience as well as that of so many other individuals and their families. I entered with the fervent belief that advances in research efforts can —and will—win the fight against epilepsy. Scientific research gives hope to patients and their loved ones, offering the very best opportunity we have to truly transform and save lives.

Our work with the epilepsy research community began by opening up a conversation to challenge what had seemed to us to be an acceptance of the status quo-a sort of complacency with the repeated seizures and intolerable treatment side effects, particularly in the $30 \%$ of patients who are deemed intractable.

Susan Axelrod

SAxelrod@CUREepilepsy.org

1 Citizens United for Research in Epilepsy, Chicago, IL, USA
We challenged that status quo and found, to our delight, great acceptance and excitement within the epilepsy research and treating community, which resulted in partnerships that have continued to this day and have been instrumental to CURE's growth and progress.

One of those earliest partnerships was with Steve White.

I first met Steve when he was Director of the Anticonvulsant Screening Program-an invaluable program that has been responsible for the development of a multitude of new therapies that have improved the lives of countless patients with epilepsy.

Although he is a basic scientist, it was clear from our first meeting what motivates Steve. There's never been a doubt that his primary focus and end goal is always the patient with epilepsy. Along with his scientific prowess, which will be addressed in other commentaries in this special issue, it is fitting to honor Steve for his winning combination of expertise and passion, and the leadership role he has played in moving the needle forward and advancing critical therapies.

The day that Steve informed me of his willingness to join CURE as our Research Director was a day I will never forget. We had just reached a point in the organization where it seemed critically important to build out our own internal scientific team and I remember exactly where I was when I learned that Steve was willing to join us in this capacity. I had never imagined that our fledgling organization could attract the likes of Steve White!

Steve joined CURE in 2011 and he did not disappoint. The accolades we so often heard about him were confirmed over and over again. He led our young, internal team with professionalism, thoughtfulness and with the end goal- the patient's well-being_always foremost in mind.

In previous years, I had come to know many of Steve's colleagues, students he had mentored, and people who 
knew and admired him for his professional accomplishments. I heard frequently of his exceptional mentorshiphow supportive, inspiring, and wise he was in this role. We owe Steve a debt of gratitude for influencing so many young scientists to enter the field of epilepsy and building the pipeline for the future.

In his new position at CURE, we also witnessed his remarkable abilities beyond those we were already cognizant of. He was able to immediately establish trust with patients and family members through respectful and sensitive interactions. He was able, with great facility, to translate challenging scientific concepts into language that was easily grasped by individuals who had had no scientific training. This is a unique and powerful ability.

As patients who admittedly have some degree of impatience with the speed of scientific discovery, we asked a lot of Steve. We asked him to always think outside the box. We asked him to challenge epilepsy researchers and traditional scientific protocols. We asked him, on occasion, to break with customary practices in the research community, many members of which were his long-time colleagues and friends.

Not only did he help us shape our research initiatives to accelerate discoveries, he did it with grace, thoughtfulness, and a seemingly innate ability to bring people together, never alienating or offending. He was always able to effortlessly bring about understanding, consensus, collaboration and a sense of communal responsibility to always strive for the best outcomes for patients.

As a family member and a patient advocate, I am thrilled to have been invited to contribute to this important tribute to Steve. His quiet but powerful leadership, his sensitivity to others, and his strong moral compass are extraordinary and deserve the highest commendation and the deepest appreciation from patients and family members worldwide. 